\title{
Kyphosis and paraspinal muscle composition in older men: a cross-sectional study for the osteoporotic fractures in men (MrOS) research group
}

Wendy B Katzman ${ }^{1 *}$, Dana Miller-Martinez ${ }^{2}$, Lynn M Marshall ${ }^{3}$, Nancy E Lane ${ }^{4}$ and Deborah M Kado ${ }^{5}$

\begin{abstract}
Background: The prevalence of hyperkyphosis is increased in older men; however, risk factors other than age and vertebral fractures are not well established. We previously reported that poor paraspinal muscle composition contributes to more severe kyphosis in a cohort of both older men and women.

Methods: To specifically evaluate this association in older men, we conducted a cross-sectional study to evaluate the association of paraspinal muscle composition and degree of thoracic kyphosis in an analytic cohort of 475 randomly selected participants from the Osteoporotic Fractures in Men (MrOS) study with baseline abdominal quantitative computed tomography (QCT) scans and plain thoracic radiographs. Baseline abdominal QCT scans were used to obtain abdominal body composition measurements of paraspinal muscle and adipose tissue distribution. Supine lateral spine radiographs were used to measure Cobb angle of kyphosis. We examined the linear association of muscle volume, fat volume and kyphosis using loess plots. Multivariate linear models were used to investigate the association between muscle and kyphosis using total muscle volume, as well as individual components of the total muscle volume, including adipose and muscle compartments alone, controlling for age, height, vertebral fractures, and total hip bone mineral density (BMD). We examined these associations among those with no prevalent vertebral fracture and those with $\mathrm{BMl}<30 \mathrm{~kg} / \mathrm{m}^{2}$.
\end{abstract}

Results: Among men in the analytic cohort, means (SD) were $74(\mathrm{SD}=5.9)$ years for age, and $37.5(\mathrm{SD}=11.9)$ degrees for Cobb angle of kyphosis. Men in the lowest tertile of total paraspinal muscle volume had greater mean Cobb angle than men in the highest tertile, although test of linear trend across tertiles did not reach statistical significance. Neither lower paraspinal skeletal muscle volume $(p$-trend $=0.08)$, or IMAT ( $p$-trend $=0.96$ ) was associated with greater kyphosis. Results were similar among those with no prevalent vertebral fractures. However, among men with $\mathrm{BMl}<30 \mathrm{~kg} / \mathrm{m}^{2}$, those in the lowest tertile of paraspinal muscle volume had greater adjusted mean kyphosis (40.0, 95\% Cl: 37.8 - 42.1) compared to the highest tertile (36.3, 95\% Cl: 34.2 - 38.4).

Conclusions: These results suggest that differences in body composition may potentially influence kyphosis. Keywords: Kyphosis, Hyperkyphosis, Spinal muscle composition, Vertebral fractures, BMI

\footnotetext{
* Correspondence: wendy.katzman@ucsfmedctr.org

'Department of Physical Therapy and Rehabilitation Science, University of California, San Francisco, CA 94158, USA

Full list of author information is available at the end of the article
} 


\section{Background}

Hyperkyphosis, an exaggerated curvature in the thoracic spine, is commonly observed in the older population although its causes have not been well investigated. Greater degrees of kyphosis impairs physical function [1-5] and well-being [6], and increases load on the vertebral bodies $[7,8]$ that potentially could increase the risk for vertebral compression fractures. A recent study confirms the need for more comprehensive assessment of health outcomes in older adults with greater degree of kyphosis [9]. Hyperkyphosis is considered a problem that primarily affects older women, even though the prevalence of hyperkyphosis in older men is estimated to range from $15-40 \%$, depending upon how kyphosis is defined $[6,10]$. While commonly recognized risk factors for hyperkyphosis in women include advanced age, low bone mass and underlying vertebral fractures [11], only $1 / 3$ of older women with the most severe kyphosis have vertebral compression fractures (VCFs) [12], suggesting other factors contribute to kyphosis that have not been well defined. Furthermore, it is not known whether men have similar risk factors for hyperkyphosis.

Recently Katzman, et al. determined that paraspinal trunk muscle composition is associated with hyperkyphosis in a cohort of older community-dwelling men and women [10]. Among individuals with a greater degree of kyphosis, there was more adipose accumulation within the paraspinal muscles. Imaging methods that include computed tomography and MRI are the most accurate methods available for quantifying skeletal muscle composition in vivo, and these methods permit measurement of skeletal muscle and adipose tissues within the muscle fascia [13]. While MRI does not expose an individual to radiation, it overestimates the amount of adipose tissue within the erector spinae muscle fascia [14], suggesting that $\mathrm{CT}$ imaging of the erector spinae may be more precise than MRI. Attenuation refers to the energy of the $\mathrm{x}$-ray beam as it passes through the tissue, and whereas all tissue attenuates the $x$-ray beam, muscle attenuates more than adipose. Attenuation indicates the density of muscle with lower attenuation indicative of greater lipid infiltration [15]. An attenuation value, called a Hounsfield Unit (HU), is assigned to each volume element (or voxel) in the CT image. Previous work demonstrated that muscle and adipose tissues have distinct HU ranges [15-18]. Thus, CT imaging permits segmentation of the total muscle volume contained within the fascial borders into its skeletal muscle and adipose tissue components.

Skeletal muscle composition changes with aging and differs according to sex. During aging, adipose tissue volume increases, skeletal muscle tissue volume declines, and the remaining muscle tissue is susceptible to lipid infiltration [19]. Younger adults have greater muscle volume and higher muscle attenuation than older adults [20]. Moreover, sex also affects muscle composition of the skeletal muscles, particularly in the paraspinal muscles, and men have higher paraspinal muscle attenuation than women $[21,22]$. Given these age-related changes in paraspinal muscle composition and the differences in paraspinal attenuation in men compared with women, further investigation of the association of paraspinal muscle composition with kyphosis in older men is warranted.

We conducted a cross-sectional study to investigate differences in degree of thoracic kyphosis according to paraspinal muscle composition in older men using data from the Osteoporotic Fractures in Men (MrOS) Study. We hypothesized that both lower paraspinal muscle volume and higher paraspinal intermuscular adipose volume would be associated with greater degree of Cobb angle of kyphosis, independent of known and potential covariates age, weight, height, abdominal fat, hip bone mineral density, and prevalent vertebral fractures. We further hypothesized that obese men would have more paraspinal muscle volume than men with $\mathrm{BMI}<30 \mathrm{~kg} / \mathrm{m}^{2}$ that would have a protective effect on Cobb angle of kyphosis, thus warranting examining these associations according to BMI.

\section{Methods}

\section{Participants}

The Osteoporotic Fractures in Men (MrOS) cohort consists of 5,994 men aged 65 or older who were recruited from 6 academic medical centers in Birmingham, AL; Minneapolis, MN; Palo Alto, CA; Pittsburgh, PA; Portland, OR; and San Diego, CA [23,24]. The primary purpose of MrOS has been to describe the risk factors for osteoporotic fractures in older men. Funding for MrOS began in July 1999 and the study is now its 13th year. At baseline, all men were referred for plain thoracic and lumbar radiographs and 3786 men were referred for quantitative computed tomography (QCT) scans of the hips, lumbar spine and abdomen. Abdominal body composition measures were performed for a random sample of 667 participants in the sample of 3786 who had been referred for QCT scans at baseline [25]. The analytic cohort for our study included the 475 randomly selected participants from the MrOS study with both baseline abdominal body composition measures from QCT scans and plain thoracic radiographs.

Approval of the conduct of the MrOS study was obtained from the Institutional Review Board of each of the participating medical centers and written informed consent was obtained from all study participants.

\section{Muscle variables}

Baseline abdominal QCT scans were used to obtain abdominal body composition measurements of abdominal 
wall muscle and adipose tissue distribution. Abdominal scans were obtained using a standardized protocol which specified scanning from the mid-L3 to the mid-L5 vertebra at $5-\mathrm{mm}$ slice thickness with participants in the supine position. We completed abdominal body composition measurements on the scan at the L4-L5 intervertebral disc level. Scans were processed by two specialists at Oregon Health and Science University (OHSU) using a standardized protocol as previously described [26]. Briefly, a spline tool was used to trace the outer and inner fascial borders of the abdominal wall musculature comprising 6 bilateral muscle groups - the rectus abdominis, external oblique, internal oblique, transverse abdominis, psoas and paraspinals. The paraspinal muscle group included the muscles on each side of the vertebral body in the lumbar region located lateral to the lumbar vertebral spinous process and posterior to the lumbar vertebral transverse processes comprising the longissimus, iliocostalis and multifidus muscles. Intermuscular adipose tissue (IMAT) included adipose tissue located around muscle bundles within each muscle group (Figure 1). Attenuation ranges used to estimate volumes $\left(\mathrm{cm}^{3}\right)$ of muscle were 0 to $100 \mathrm{HU}$ $[15,18]$ and of IMAT were -190 to $-30 \mathrm{HU}[16,17]$. Thus, for all muscle groups studied, we had three volume variables: skeletal muscle only, IMAT, and total volume which is the sum of skeletal muscle plus IMAT volume. Muscle attenuation (HU) was obtained for the total volume and the skeletal muscle only volume. To account for variability in image attenuation across scanners, the $\mathrm{HU}$ in the scans were scaled to the

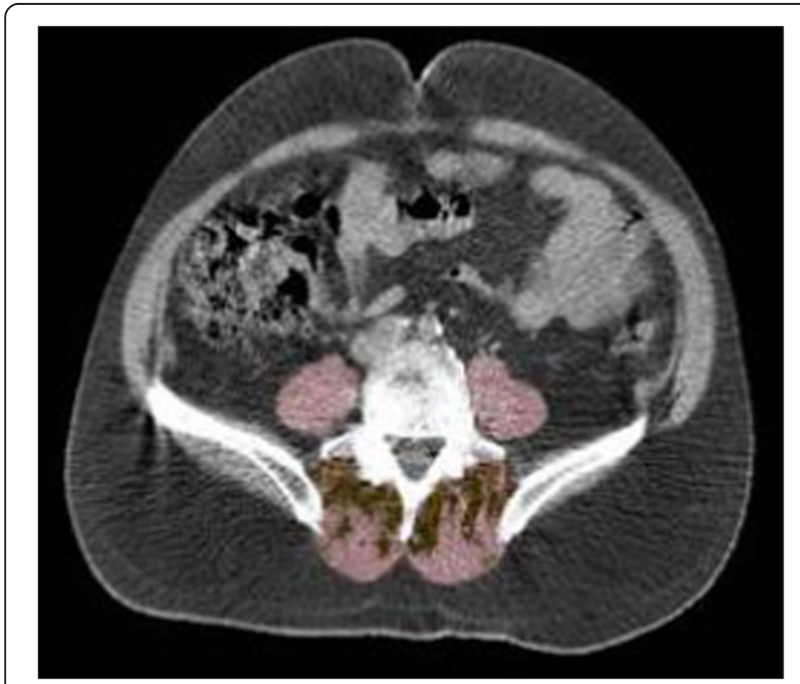

Figure 1 Paraspinal muscle group including the longissimus, iliocostalis and multifidus muscles (posterior to the vertebral body) and psoas muscle (lateral to the vertebral body) with intermuscular adipose tissue (IMAT) shown at L4/L5 interspace by abdominal QCT. Pink= muscle tissue; yellow = IMAT. calibration standard for water density $\left(0 \mathrm{mg} / \mathrm{cm}^{3}\right)$. Interand intra-rater reliability was monitored throughout image processing with intraclass correlation coefficients (ICC). Final intra-rater ICCs for all measures were $\geq 0.98$ for rater 1 and $\geq 0.95$ for rater 2 , and final inter-rater ICCs were all $\geq 0.96$.

\section{Cobb angle of kyphosis measurements}

The Cobb angle of kyphosis was measured from supine lateral spine radiographs taken during baseline visit 1 . We used the modified Cobb method [27] with a fixed cut-off of T4 and T12, largely because T1 to T3 are usually not well visualized on lateral spine films due to the overlying shoulders and scapulae interfering with the projection. Technicians placed 6 points corresponding to the 4 corners of the vertebral body and the midpoints of the endplates on each vertebral body from T4 to T12. From the superior surface of T4 and inferior surface of T12, a computerized digitization program erected perpendicular lines whose intersection was the kyphotic angle (Figure 2). If for any reason T4 or T12 were not visible, the next adjacent visible vertebra ( $\mathrm{T} 5$ if $\mathrm{T} 4$ not visible or $\mathrm{T} 11$ if $\mathrm{T} 12$ not visible) was used as an alternative. The ICC for Cobb angle of kyphosis has been previously reported 0.99 [27].

\section{Vertebral fracture measurements}

Vertebral fractures were adjudicated from baseline lateral lumbar and thoracic spine radiographs based upon a

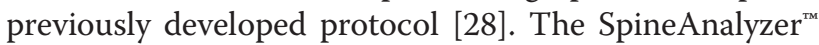
(Optasia Medical Ltd., Cheadle, UK) workflow tool was used to automate placement of 6-point morphometric points on each radiograph in order to efficiently identify the vertebral bodies for evaluation. An expert radiologist then graded each vertebra as normal (0), mild (1), moderate (2), severe (3) using the well-established, highly reliable semi-quantitative Genant method [29]. Prevalent vertebral fractures were defined as grade 2 or 3 .

\section{Anthropometric measurements}

Body weight and height were taken at baseline by an examiner using standard equipment, including a Harpenden stadiometer and balance beam scale while wearing indoor street clothing without shoes [23]. Body mass index (BMI) was calculated as weight divided by the square of height in meters $\left(\mathrm{kg} / \mathrm{m}^{2}\right)$. Bone mineral density (BMD) was also measured at baseline in the proximal femur and lumbar spine using DXA measured by Hologic QDR 4500 densitometers.

\section{Statistical analysis}

We built models to control for potential confounding using an empirical approach. We examined the association between an a priori group of potential confounders 


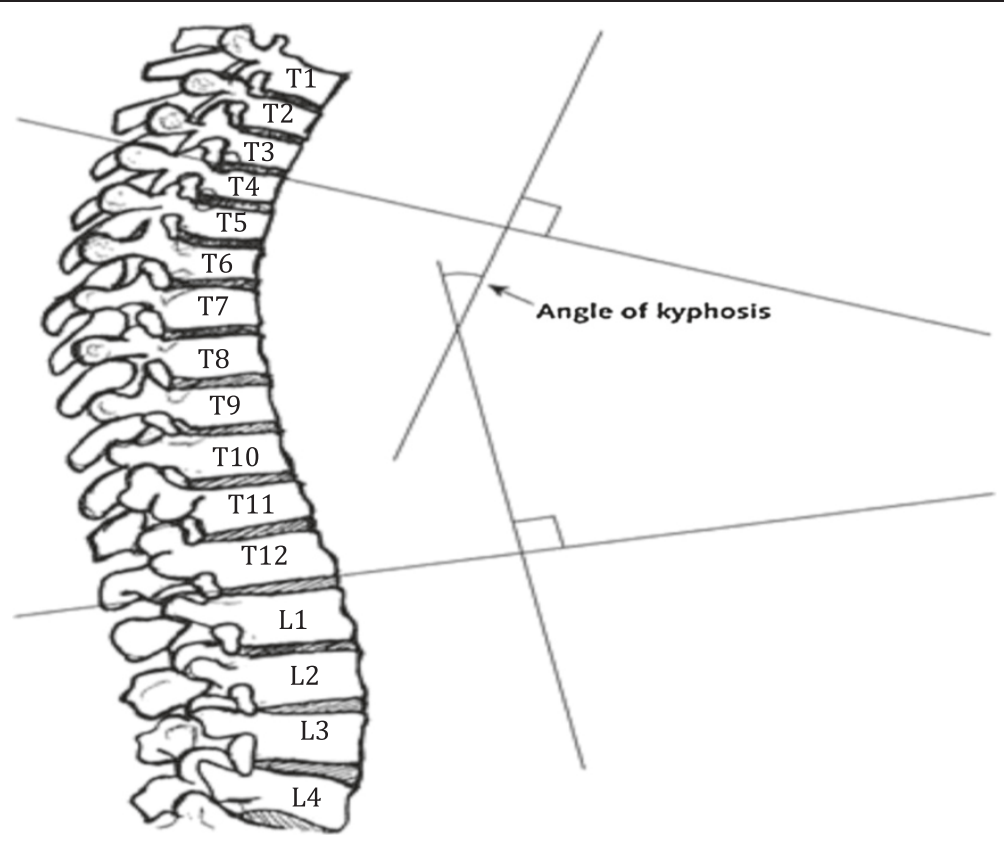

Figure 2 Cobb angle of kyphosis, measured as the angle of intersecting lines drawn from the superior endplate of T4 and the inferior endplate of $\mathrm{T} 12$, calculated from a radiographic image shown.

and Cobb angle and based on the change in the regression coefficient, we used $\mathrm{p}$ value $<0.1$ to select confounders including age, height, vertebral fractures, and total hip bone mineral density (BMD). Loess plots were used initially to determine the shape of the relations of muscle volume, adipose volume and Cobb angle. Because associations were not linear, muscle mass measurements were divided into quintiles, quartiles and tertiles to investigate non-linear trends in kyphosis, but due to the limited sample size, we used tertiles for our analysis. Multivariate linear models were used to investigate the association between the muscle variables and kyphosis using total muscle volume, as well as individual components of the total muscle volume that included the IMAT and skeletal muscle compartments alone. All multivariable analyses controlled for age, height, vertebral fractures, and total hip BMD. These associations were also investigated separately for the total abdominal (rectus abdominis, transversus abdominis, internal obliques and external obliques), psoas and paraspinal muscle groups. We examined these associations among those with no prevalent vertebral fracture $(n=429)$ and among those with $\mathrm{BMI}<30 \mathrm{~kg} / \mathrm{m}^{2} \quad(\mathrm{n}=394)$. We conducted sensitivity analyses with different adjustment variables as well as an alternate definition of hyperkyphosis $>40$ degrees.

\section{Results}

The subject characteristics of the overall MrOS cohort, means (standard deviation), were $74(\mathrm{SD}=5.9)$ years for age, $174(\mathrm{SD}=6.8) \mathrm{cms}$ for height, $0.96(\mathrm{SD}=0.14) \mathrm{g} / \mathrm{cm}^{2}$ for total hip BMD, $27(\mathrm{SD}=3.8) \mathrm{kg} / \mathrm{m}^{2}$ for BMI, and $7.5 \%$ had prevalent vertebral fractures. In the analytic cohort of 475 men, means $(\mathrm{SD})$ were $74(\mathrm{SD}=5.9)$ years for age and $37.5(\mathrm{SD}=11.9)$ degrees for Cobb angle of kyphosis (Table 1). Men with $\mathrm{BMI}<30 \mathrm{~kg} / \mathrm{m}^{2}$ were older, had greater Cobb angle, lower hip BMD, and lower total abdominal muscle volume, but no difference in total paraspinal or total psoas muscle volume. When we separated total muscle volume into its skeletal muscle tissue and IMAT components, skeletal muscle volume but not attenuation in all of the abdominal wall muscle groups was greater, and IMAT in the abdominal and psoas muscle groups was lower in men with $\mathrm{BMI}<30 \mathrm{~kg} / \mathrm{m}^{2}$.

Men in the lowest tertile of total paraspinal muscle volume had greater mean Cobb angle than men in the highest tertile (Table 2), although the test of linear trend across tertiles did not reach statistical significance. When we further separated total paraspinal volume into its skeletal muscle tissue and IMAT components, neither lower paraspinal skeletal muscle volume ( $\mathrm{p}$-trend $=0.08$ ), or IMAT ( $\mathrm{p}$-trend $=0.96$ ) was associated with greater kyphosis. Results were similar among those with no prevalent vertebral fractures. However, among men with $\mathrm{BMI}<30 \mathrm{~kg} / \mathrm{m}^{2}$, those in the lowest tertile of paraspinal skeletal muscle volume had greater adjusted mean kyphosis (40.0, 95\% CI: 37.8 - 42.1) compared to men in the highest tertile (36.3, 95\% CI: 34.2 - 38.4). There were no associations of the total abdominal or total psoas muscle volumes with Cobb angle of 
Table 1 Baseline characteristics of MrOs sample $(n=475)$

\begin{tabular}{|c|c|c|c|c|}
\hline & \multirow[t]{2}{*}{ Full sample mean (SD) } & \multicolumn{3}{|c|}{ Stratified by BMI mean (SD) } \\
\hline & & $\mathrm{BMI}<30(\mathrm{n}=399)$ & $\mathrm{BMI} \geq 30(n=76)$ & $p$-value for difference ${ }^{a}$ \\
\hline Age (years) & $74.2(5.86)$ & $74.7(5.86)$ & $71.6(5.09)$ & $<.001$ \\
\hline Cobb angle of kyphosis (degrees) & $37.5(11.90)$ & $37.9(12.0)$ & $35.2(11.3)$ & .07 \\
\hline Height (cm) & $173.6(6.97)$ & $173.7(7.19)$ & $172.9(5.72)$ & .28 \\
\hline Total hip BMD $\left(\mathrm{g} / \mathrm{cm}^{2}\right)$ & $0.94(0.14)$ & $0.92(0.14)$ & $1.01(0.13)$ & $<.001$ \\
\hline BMI $\left(\mathrm{kg} / \mathrm{m}^{2}\right)$ & $26.8(3.21)$ & $25.8(2.37)$ & $32.0(1.54)$ & $<.001$ \\
\hline Prevalent vertebral fracture (\% yes) & 9.68 & 10.5 & 5.26 & 0.16 \\
\hline Total paraspinal muscle volume $\left(\mathrm{cm}^{3}\right)$ & $18.6(3.79)$ & $18.7(3.69)$ & $18.0(4.28)$ & 0.15 \\
\hline Paraspinal muscle volume $\left(\mathrm{cm}^{3}\right)$ & $12.1(3.47)$ & $12.3(3.42)$ & $11.4(3.64)$ & 0.04 \\
\hline Paraspinal muscle attenuation (HU) & $43.6(5.48)$ & $43.5(5.40)$ & $43.9(5.91)$ & 0.57 \\
\hline Paraspinal adipose tissue volume $\left(\mathrm{cm}^{3}\right)$ & $6.41(2.61)$ & $6.36(2.65)$ & $6.62(2.37)$ & 0.43 \\
\hline Total psoas muscle volume $\left(\mathrm{cm}^{3}\right)$ & $11.1(2.65)$ & $11.1(2.58)$ & $10.7(2.98)$ & 0.15 \\
\hline Psoas muscle volume $\left(\mathrm{cm}^{3}\right)$ & $10.0(3.16)$ & $10.2(2.99)$ & $8.82(3.76)$ & .003 \\
\hline Psoas muscle attenuation (HU) & $51.53(5.13)$ & $51.64(5.43)$ & $50.98(3.05)$ & 0.14 \\
\hline Psoas adipose tissue volume $\left(\mathrm{cm}^{3}\right)$ & $1.07(0.91)$ & $0.92(0.77)$ & $1.85(1.15)$ & $<.001$ \\
\hline Total abdominal muscle volume $\left(\mathrm{cm}^{3}\right)$ & $63.9(8.11)$ & $63.5(7.82)$ & $65.8(9.29)$ & .048 \\
\hline Abdominal muscle volume $\left(\mathrm{cm}^{3}\right)$ & $48.3(10.3)$ & $48.9(9.82)$ & $45.2(12.0)$ & .01 \\
\hline Abdominal muscle attenuation (HU) & $45.79(4.36)$ & $45.84(4.54)$ & $45.56(3.30)$ & 0.53 \\
\hline Abdominal adipose tissue volume $\left(\mathrm{cm}^{3}\right)$ & $15.6(6.01)$ & $14.6(5.50)$ & $20.6(6.14)$ & $<.001$ \\
\hline
\end{tabular}

${ }^{\mathrm{a}} \mathrm{p}$-value represents $t$-test (for continuous variables) or Chi-square test (for categorical variables) for difference between those with $\mathrm{BMI}<30 \mathrm{~kg} / \mathrm{m}^{2}$ versus those with $\mathrm{BMI} \geq 30 \mathrm{~kg} / \mathrm{m}^{2}$.

$\mathrm{cm}=$ centimeters $; \mathrm{cm}^{3}=$ centimeters ${ }^{3} ; \mathrm{g} / \mathrm{cm}^{2}=$ grams $/$ centimeter ${ }^{2} ; \mathrm{kg} / \mathrm{m}^{2}=$ kilograms $/ \mathrm{meter}^{2} ; \mathrm{HU}=$ Hounsfield units.

kyphosis (Table 3), and no significant associations of the adjusted mean Cobb angle of kyphosis according to abdominal and psoas skeletal muscle and IMAT volumes, $\mathrm{p}>.05$ (data not shown).

The sensitivity analysis where we replaced paraspinal IMAT with paraspinal muscle attenuation in models that also included paraspinal muscle volume showed similar findings. Specifically, neither paraspinal skeletal muscle volume or paraspinal muscle attenuation showed significant associations with Cobb angle, with the exception of paraspinal muscle volume among those with men with $\mathrm{BMI}<30$ (data not shown). Consistent with the results in Table 2, among men with $\mathrm{BMI}<30 \mathrm{~kg} / \mathrm{m}^{2}$, having less paraspinal muscle volume is associated with greater Cobb angle. In the multivariate adjusted model, when Cobb angle was dichotomized $>40$ degrees, there was no association with paraspinal muscle volume or adipose volume, $\mathrm{p}>0.05$ (data not shown).

\section{Discussion}

We found that paraspinal muscle volume influenced kyphosis among those with $\mathrm{BMI}<30 \mathrm{~kg} / \mathrm{m}^{2}$. Lower paraspinal muscle volume may reduce the capacity of paraspinal muscles to extend and stabilize the spine, resulting in increased kyphosis. In contrast, muscle volume in the abdominal and psoas muscles that predominantly flex the spine, did not influence kyphosis. Furthermore, we found that paraspinal muscle volume, rather than paraspinal muscle attenuation, a measure of intramuscular adipose tissue, had greater influence on kyphosis than IMAT in our cohort of older men.

These results are in contrast to our previous finding among older men and women in the Health Aging and Body Composition (Health ABC) Study that paraspinal muscle attenuation, not muscle volume, is associated with kyphosis. It is possible these differences could be because the Health $A B C$ Study included men and women, and our MrOS cohort included men only who have greater muscle volume and attenuation than women. Our results could also be explained by differences in image acquisition and processing of the computed tomography scans that were used to obtain the muscle composition measurements; there are no standard methods, making comparisons of the Health $\mathrm{ABC}$ and MrOS findings difficult. Another hindrance to comparing results is that in our previously reported Health $\mathrm{ABC}$ study, muscle volume was categorized as the volume of non-bone, nonadipose tissue within the fascial plane of the specified muscle groups, whereas in our current MrOS study, muscle volume was defined as muscle and adipose tissue within the plane of the fascia inclusive of intramuscular adipose tissue. Furthermore, 
Table 2 Adjusted mean Cobb angle of kyphosis according to volumes of paraspinal muscle and intermuscular adipose tissue (IMAT) among 475 men aged $\geq 65$ years

$$
\text { Least square mean }(95 \% \mathrm{Cl}) \text { for Cobb angle of kyphosis (degrees) }
$$
within tertile of muscle measure

Muscle volume tertile (from lowest to highest)

1 2 3

Full analytic sample ${ }^{a}$

MODEL 1

Total paraspinal muscle volume(includes paraspinal muscle and IMAT volume combined)

MODEL 2

Paraspinal Muscle Volume

Among those with no prevalent vertebral fracture ${ }^{b}$

MODEL 1

Total paraspinal muscle volume (includes paraspinal muscle and IMAT volume combined)

$$
37.5(35.6,39.4) \quad 37.8(35.9,39.6) \quad 35.9(33.9,37.9)
$$

MODEL 2

Paraspinal Muscle Volume

MODEL 1

Total paraspinal muscle volume (includes paraspinal muscle and IMAT volume combined)

MODEL 2

Paraspinal Muscle Volume

a Least square means and $95 \% \mathrm{Cl}$ estimated from multiple linear regression adjusted for age, height, prevalent vertebral fracture, and total hip BMD with paraspinal muscle volume and paraspinal IMAT in the same model. bLeast square means and $95 \%$ Cl estimated from multiple linear regression adjusted for age, height, and total hip BMD with paraspinal muscle volume and paraspinal IMAT in the same model.

$\mathrm{kg} / \mathrm{m}^{2}=$ kilograms $/$ meter $^{2}$.

Table 3 Adjusted mean Cobb angle of kyphosis according to volumes of abdominal muscle and psoas muscle among 475 men aged $\geq 65$ years*

\section{Least square mean $(95 \% \mathrm{Cl})$ for Cobb angle of kyphosis (degrees)} within tertile of muscle measure

Muscle volume tertile (from lowest to highest)

1

1

Full analytic sample

Total abdominal muscle volume

$36.6(34.7,38.4)$

$38.8(37.0,40.7)$

3

Total psoas muscle volume

$37.6(35.8,39.5)$

$38.0(36.2,39.8)$

$37.1(35.2,39.0)$

$36.9(35.0,38.7)$

Among those with no prevalent vertebral fracture ${ }^{b}$

Total abdominal muscle volume

Total psoas muscle volume

Among those with $\mathrm{BMI}<30^{\mathrm{a}}$

Total abdominal muscle volume

Total psoas muscle volume

$\begin{array}{ll}36.7(34.7,38.6) & 38.0(36.1,39.8) \\ 37.5(35.6,39.5) & 37.5(35.6,39.3)\end{array}$

$38.0(36.1,39.8)$
$37.5(35.6,39.3)$

$36.6(34.6,38.5)$

0.94

$36.2(34.3,38.1)$

0.32

$$
37.4(35.3,39.4)
$$

$39.0(37.0,40.9)$

$37.3(35.1,39.5)$

0.97

$38.5(36.4,40.6)$

*Total abdominal muscle volume and total psoas muscle volume are tested in separate models.

aLeast square means and $95 \% \mathrm{Cl}$ estimated from multiple linear regression adjusted for age, height, prevalent vertebral fracture, and total hip BMD. ${ }^{b}$ Least square means and $95 \% \mathrm{Cl}$ estimated from multiple linear regression adjusted for age, height, and total hip BMD. 
IMAT was not measured or included in the Health ABC analysis, whereas we controlled for IMAT in our analyses. However, when we did investigate paraspinal muscle attenuation in MrOS, it was not associated with Cobb angle of kyphosis. When we performed a sensitivity analysis and replaced IMAT with paraspinal muscle attenuation, the results did not appreciably change. In addition, while we used a continuous measure of Cobb angle of kyphosis in MrOS, in Health $\mathrm{ABC}$ we used a dichotomous variable of hyperkyphosis defined as $>40$ degrees, which could reflect a threshold effect of paraspinal muscle attenuation on Cobb angle of kyphosis. Nonetheless, we found no evidence of a threshold effect within the MrOS cohort. Finally, Health ABC measurements of Cobb angle were obtained from supine computed tomography scout scans, whereas in MrOS we obtained these measurements from supine lateral spine radiographs, and we do not know how well these measurements compare.

Vertebral fractures, commonly thought to be the cause of excessive kyphosis, did not influence the association between paraspinal muscle volume, paraspinal IMAT and Cobb angle of kyphosis. These results provide further evidence that factors other than vertebral fractures are responsible for greater degree of kyphosis. We found that paraspinal muscle volume, a factor that is potentially modifiable with exercise, is associated with Cobb angle of kyphosis in our cohort of older men with $\mathrm{BMI}<30 \mathrm{~kg} / \mathrm{m}^{2}$ versus high BMI.

Whereas decreased paraspinal muscle attenuation, not muscle volume, has been associated with decline in physical performance among older adults [30,31], in our study, we found reduced paraspinal muscle volume, not muscle attenuation, influenced kyphosis. Resistance exercise trials targeting lower extremity muscle groups in older adults have reported that highintensity resistance exercise produces large increase in muscle strength [32,33]. Improvement in lower extremity muscle strength following high intensity strengthening has also been associated with increased muscle volume and muscle attenuation [32-36]. Several randomized controlled trials of exercise have included back extensor muscle strengthening to reduce kyphosis [37-41], and it is possible that strengthening the back extensor muscles could improve paraspinal muscle volume and attenuation. Unfortunately the effects of back extensor muscle strengthening on paraspinal muscle volume or attenuation have not been investigated, and it is not known whether improving paraspinal muscle volume or attenuation reduces kyphosis.

While the phenotype of a smaller-framed older woman with hyperkyphosis is well-recognized, our findings suggest this phenotype also exists in older men. Body mass index, a proxy for human body fat based on an individual's weight and height, influenced kyphosis in our cohort of men. We found $83 \%$ of the men had BMI $<30 \mathrm{~kg} / \mathrm{m}^{2}$, and among these men, low paraspinal muscle volume was associated with greater Cobb angle of kyphosis. In fact, among those with $\mathrm{BMI}<30 \mathrm{~kg} / \mathrm{m}^{2}$ and in the lowest tertile of paraspinal muscle volume, the mean kyphosis was 40.0 degrees (95\% CI $=37.8-42.1$ ), the threshold for defining hyperkyphosis in older adults $[42,43]$. Hyperkyphosis, low paraspinal muscle volume and $\mathrm{BMI}<30 \mathrm{~kg} / \mathrm{m}^{2}$ may reflect an underlying geriatric syndrome that impacts both older men and women. This kyphotic phenotype may place older men at risk for vertebral compression fractures due to the increased spinal load and impaired functional outcomes that are associated with greater kyphosis. While obesity did appear to have a protective effect on kyphosis in our cohort, intraorgan fat has been associated with poor health outcomes in older adults [44]. We are not advocating for obesity as a means to control excessive kyphosis, rather the need for more comprehensive assessment of health status among older adults with hyperkyphosis.

\section{Limitations}

This study has a number of strengths including that the MrOS cohort includes community-dwelling older men residing in multiple geographic areas of the United States who were not preselected for hyperkyphosis. The kyphosis and the paraspinal muscle measurements were assessed at baseline with high reliability in a subgroup of study subjects. However, this study also has a number of limitations including possible misclassification of muscle measurements depending upon the exact location of the QCT scan slice. The paraspinal muscle group does increase in size closer its attachment sites, therefore our paraspinal muscle volume estimates measured at the L4L5 intervertebral disc space may be underestimated, as would any association of paraspinal muscle volume and kyphosis found in this study. Another potential limitation is that we could not measure muscle volumes in the thoracic spine due to the CT technology used. However, our results suggest that lumbar paraspinal muscles serve as a proxy for muscle degeneration in the upper spine as well. We also used BMD measurements from the hip even though the muscle and kyphosis measurements were made in the spine. Hip BMD is often used in place of spine BMD because there is less artifact in the hip and the lumbar spine BMD from DXA can be overestimated because of extravertebral calcification (e.g. osteophytes). Hip BMD thus provides a more accurate measurement, particularly in elderly subjects. Moreover, when we replaced hip BMD with volumetric measurements of spine BMD with QCT, there was no appreciable difference in the results. The MrOS population 
may have degenerative spine conditions that could increase kyphosis and our regression analysis did not include adjustment for these degenerative changes. However, this is beyond the scope of this study. We chose to measure global Cobb angle using T4 and T12 endplates rather than other methods such as the centroid method that is less affected by endplate tilt [45] because the global method using the endplates of T4 and T12 is reliable and one of the most commonly used radiographic methods for measuring thoracic kyphosis. In future studies, we may consider using other methods that reflect changes in the middle of the curve. Finally, supine measurements of Cobb angle of kyphosis underestimate the degree of kyphosis compared to standing measurements, particularly among those with greater degree of kyphosis [27]. However, this would serve to underestimate any effects we found.

\section{Conclusions}

In summary, we found that paraspinal muscle volume influenced kyphosis among older men with $\mathrm{BMI}<30 \mathrm{~kg} / \mathrm{m}^{2}$, and lower paraspinal muscle volume was associated with greater degree of thoracic kyphosis. Interestingly, prevalent vertebral fractures commonly thought to be the cause of excessive kyphosis, did not influence the association between paraspinal muscle volume, paraspinal IMAT and Cobb angle of kyphosis. Additional studies are now warranted that evaluate if low paraspinal muscle volume contributes to worsening of kyphosis in this population.

\begin{abstract}
Abbreviations
MrOS: Osteoporotic Fractures in Men; QCT: Quantitative computed tomography; BMD: Bone mineral density; SD: Standard deviation; IMAT: Intermuscular adipose tissue; BMI: Body mass index; $\mathrm{kg} / \mathrm{m}^{2}$ : Kilograms/ meter $^{2}$; HU: Hounsfield Unit; Cl: Confidence interval; Health ABC: Health Aging and Body Composition Study; OHSU: Oregon Health and Science University; cm: Centimeter; ICC: Intraclass correlation coefficients.
\end{abstract}

\section{Competing interests}

The authors declare that they have no competing interests.

\section{Authors' contributions}

WK conceived of the study, participated in its design and drafted the manuscript. DM carried out the statistical analysis. LM conceived of the study, participated in its design, drafted sections and edited the manuscript. NL participated in study design and edited the manuscript. DK conceived of the study, participated in study design and edited the manuscript. All authors read and approved the final manuscript.

\section{Acknowledgements}

This research was supported by the UCSF-Kaiser Building Interdisciplinary Research Careers in Women's Health (BIRCWH), the National Institute of Child Health and Human Development and the Office of Research on Women's Health 5 K12 HD052163. The Osteoporotic Fractures in Men (MrOS) Study is supported by National Institutes of Health funding. The following institutes provide support: the National Institute of Arthritis and Musculoskeletal and Skin Diseases (NIAMS), the National Institute on Aging (NIA), the National Center for Research Resources (NCRR), and NIH Roadmap for Medical Research under the following grant numbers: U01 AR45580, U01 AR45614, U01 AR45632, U01 AR45647, U01 AR45654, U01 AR45583, U01 AG18197, U01-AG027810, and UL1 RR024140. Other grants supporting this research are RO1 AG041921, R01 AR06828, R01 AR35582, R01 AR35583, R01 AR35584, K24
AR04884, R01 AR052000, P50 AR063043, P50 AR060752, P60 AR05473101. R21 DK066224, RO1 HL089467, and UL1 RR024140 and the Oregon Medical Research Foundation.

\section{Author details}

${ }^{1}$ Department of Physical Therapy and Rehabilitation Science, University of California, San Francisco, CA 94158, USA. ${ }^{2}$ UCLA David Geffen School of Medicine, Division of Geriatrics, University of California, Los Angeles, USA. ${ }^{3}$ Oregon Health and Science University, School of Medicine, Orthopaedics \& Rehabilitation Department, Portland, OR, USA. ${ }^{4}$ Department of Internal Medicine, University of California, Davis, CA, USA. ${ }^{5}$ Departments of Family and Preventive Medicine and Internal Medicine, University of California, San Diego, CA, USA.

Received: 2 August 2013 Accepted: 14 January 2014

Published: 16 January 2014

\section{References}

1. Hirose D, et al: Posture of the trunk in the sagittal plane is associated with gait in community-dwelling elderly population. Clin Biomech (Bristol, Avon) 2004, 19(1):57-63.

2. Ryan SD, Fried LP: The impact of kyphosis on daily functioning. J Am Geriatr Soc 1997, 45(12):1479-1486.

3. Huang MH KW, Cummings SR, Kado DM: Hyperkyphosis and decline in functional status in older community dwelling women: The Study of Osteoporotic Fractures in ASBMR. 2010.

4. Katzman WB, et al: Kyphosis and Decline in physical function over 15 years in older community-dwelling women: the study of osteoporotic fractures. J Gerontol A Biol Sci Med Sci 2013, 68(8):976-983.

5. Kado DM, et al: Hyperkyphotic posture and poor physical functional ability in older community-dwelling men and women: the Rancho Bernardo study. J Gerontol A Biol Sci Med Sci 2005, 60(5):633-637.

6. Takahashi T: Trunk deformity is associated with a reduction in outdoor activites of daily living and life satisfaction in community-dwelling older people. Osteoporos Int 2005, 16:273-279.

7. Briggs AM, et al: Thoracic kyphosis affects spinal loads and trunk muscle force. Phys Ther 2007, 87(5):595-607.

8. Bruno AG, et al: The effect of thoracic kyphosis and sagittal plane alignment on vertebral compressive loading. J Bone Miner Res 2012, 27(10):2144-2151.

9. Bayliss $M$, et al: A conceptual and disease model framework for osteoporotic kyphosis. Osteoporos Int 2013, 24(9):2423-2432.

10. Katzman W, et al: Association of Spinal Muscle Composition and Prevalence of Hyperkyphosis in Healthy Community-Dwelling Older Men and Women. J Gerontol A Biol Sci Med Sci 2012, 67(2):191-195.

11. Kado D, et al: Factors associated with kyphosis progression in older women: 15 years experience in the Study of Osteoporotic Fractures. J Bone Miner Res 2013, 28(1):179-187.

12. Kado DM, et al: Factors associated with kyphosis progression in older women: 15 years' experience in the study of osteoporotic fractures. J Bone Miner Res 2013, 28(1):179-187.

13. Ropponen A, Videman T, Battie MC: The reliability of paraspinal muscles composition measurements using routine spine MRI and their association with back function. Man Ther 2008, 13(4):349-356

14. Rossi A, et al: Quantification of intermuscular adipose tissue in the erector spinae muscle by MRI: agreement with histological evaluation. Obesity (Silver Spring) 2010, 18(12):2379-2384.

15. Goodpaster BH, et al: Skeletal muscle attenuation determined by computed tomography is associated with skeletal muscle lipid content J Appl Physiol 2000, 89(1):104-110.

16. Kvist $\mathrm{H}$, et al: Adipose tissue volume determination in males by computed tomography and $40 \mathrm{~K}$. Int J Obes 1988, 12(3):249-266.

17. Rossner $\mathrm{S}$, et al: Adipose tissue determinations in cadavers-a comparison between cross-sectional planimetry and computed tomography. Int J Obes 1990, 14(10):893-902

18. Sjostrom L: A computer-tomography based multicompartment body composition technique and anthropometric predictions of lean body mass, total and subcutaneous adipose tissue. Int J Obes 1991, 15(Suppl 2):19-30 
19. Goodpaster $\mathrm{BH}$, et al: The loss of skeletal muscle strength, mass, and quality in older adults: the health, aging and body composition study. J Gerontol A Biol Sci Med Sci 2006, 61(10):1059-1064.

20. Goodpaster $B H$, et al: Attenuation of skeletal muscle and strength in the elderly: The health ABC study. J App/ Physio/ 2001, 90(6):2157-2165.

21. Imamura $K$, et al: Human major psoas muscle and sacrospinalis muscle in relation to age: a study by computed tomography. J Gerontol 1983, 38(6):678-681

22. Anderson $\mathrm{DE}$, et al: Variations of $\mathrm{CT}$-based trunk muscle attenuation by age, sex, and specific muscle. J Gerontol A Biol Sci Med Sci 2013, 68(3):317-323

23. Orwoll E, et al: Design and baseline characteristics of the osteoporotic fractures in men (MrOS) study-a large observational study of the determinants of fracture in older men. Contemp Clin Trials 2005, 26(5):569-585.

24. Blank JB, et al: Overview of recruitment for the osteoporotic fractures in men study (MrOS). Contemp Clin Trials 2005, 26(5):557-568.

25. Miljkovic l, et al: Abdominal myosteatosis is independently associated to hyperinsulinemia and insulin resistance among older men without diabetes. Obesity (Silver Spring) 2013, 21(10):2118-2125.

26. Marshall LM, et al: Dimensions and volumetric BMD of the proximal femur and their relation to age among older U.S. men. J Bone Miner Res 2006, 21(8):1197-1206.

27. Kado DM, Christianson RN, Palermo L, Smith-Bindman R, Cummings SR, Greendale $\mathrm{G}$ : Comparing a supine radiographic versus standing clinical measurement of kyphosis in older women: the fracture intervention trial. Spine 2006, 31(4):463-467.

28. Schousboe JT, Rosen HR, Vokes TJ, Cauley JA, Cummings SR, Nevitt MC, Black DM, Orwoll ES, Kado DM, Ensrud KE: Prediction Models of Prevalent Radiographic Vertebral Fractures Among Older Men. J Clin Densitom 2013. doi:10.1016/j.jocd.2013.09.020.

29. Genant HK, et al: Vertebral fracture assessment using a semiquantitative technique. J Bone Miner Res 1993, 8(9):1137-1148.

30. Hicks GE, et al: Trunk muscle composition as a predictor of reduced functional capacity in the health, aging and body composition study: the moderating role of back pain. J Gerontol A Biol Sci Med Sci 2005, 60(11):1420-1424.

31. Hicks GE, et al: Cross-sectional associations between trunk muscle composition, back pain, and physical function in the health, aging and body composition study. J Gerontol A Biol Sci Med Sci 2005, 60(7):882-887.

32. Binder EF, et al: Effects of progressive resistance training on body composition in frail older adults: results of a randomized, controlled trial. J Gerontol A Biol Sci Med Sci 2005, 60(11):1425-1431.

33. Fiatarone $M A$, et al: High-intensity strength training in nonagenarians. Effects on skeletal muscle. JAMA 1990, 263(22):3029-3034.

34. Frontera WR, et al: Strength conditioning in older men: skeletal muscle hypertrophy and improved function. J Appl Physiol 1988, 64(3):1038-1044.

35. Taaffe DR, et al: Alterations in muscle attenuation following detraining and retraining in resistance-trained older adults. Gerontology 2009, 55(2):217-223.

36. Taaffe DR, et al: Comparative effects of high- and low-intensity resistance training on thigh muscle strength, fiber area, and tissue composition in elderly women. Clin Physiol 1996, 16(4):381-392.

37. Bennell $\mathrm{KL}$, et al: Effects of an exercise and manual therapy program on physical impairments, function and quality-of-life in people with osteoporotic vertebral fracture: a randomised, single-blind controlled pilot trial. BMC Musculoskelet Disord 2010, 17(11). doi:10.1186/1471-2474-11-36.

38. Bautmans I, et al: Rehabilitation using manual mobilization for thoracic kyphosis in elderly postmenopausal patients with osteoporosis. J Rehabil Med 2010, 42(2):129-135.

39. Benedetti $M G$, et al: Effects of an adapted physical activity program in a group of elderly subjects with flexed posture: clinical and instrumental assessment. J Neuroeng Rehabil 2008, 5:32.

40. Greendale GA, et al: Yoga Decreases Kyphosis in Senior Women and Men with Adult-Onset Hyperkyphosis: Results of a Randomized Controlled Trial. J Am Geriatr Soc 2009, 57(9):1569-1579.

41. Itoi $E$, Sinaki M: Effect of back-strengthening exercise on posture in healthy women 49 to 65 years of age. Mayo Clin Proc 1994, 69(11):1054-1059.

42. Fon $G$, Pitt $M$, Thies A: Thoracic kyphosis: Range in normal subjects. AJR 1980, 134:979-983.
43. Voutsinas SA, MacEwen GD: Sagittal profiles of the spine. Clin Orthop 1986, 210:235-242.

44. Gastaldelli A, Basta G: Ectopic fat and cardiovascular disease: what is the link? Nutr Metab Cardiovasc Dis 2010, 20(7):481-490.

45. Briggs AM, et al: Radiographic measures of thoracic kyphosis in osteoporosis: Cobb and vertebral centroid angles. Skeletal Radiol 2007 36(8):761-767.

doi:10.1186/1471-2474-15-19

Cite this article as: Katzman et al:: Kyphosis and paraspinal muscle composition in older men: a cross-sectional study for the osteoporotic fractures in men (MrOS) research group. BMC Musculoskeletal Disorders 2014 15:19.

\section{Submit your next manuscript to BioMed Central and take full advantage of:}

- Convenient online submission

- Thorough peer review

- No space constraints or color figure charges

- Immediate publication on acceptance

- Inclusion in PubMed, CAS, Scopus and Google Scholar

- Research which is freely available for redistribution 